\title{
Reduced Dose with Maintained Image Quality Utilizing 100 kVp Carotid CT Angiography
}

\author{
Patricia Jo ${ }^{1}$, David A Leswick ${ }^{2, *}$, Derek A Fladeland ${ }^{2}$, Robert Otani², Hyun J Lim ${ }^{3}$ \\ ${ }^{1}$ College of Medicine, Department of Diagnostic Imaging, University of Calgary, Calgary, T2N 2T9, Canada \\ ${ }^{2}$ College of Medicine, Department of Medical Imaging, Royal University Hospital, University of Saskatchewan, S7N 0W8, Canada \\ ${ }^{3}$ College of Medicine, Department of Community Health \& Epidemiology, University of Saskatchewan, S7N 5E5, Canada \\ *Corresponding Author: david.leswick@saskatoonhealthregion.ca
}

Copyright $(2013$ Horizon Research Publishing All rights reserved.

\begin{abstract}
The purpose of this study is to prospectively compare image quality and radiation dose using 100- and $120-\mathrm{kVp}$ techniques for carotid $\mathrm{CT}$ angiography. Forty consecutive patients were alternated between the $100 \mathrm{kVp}$ group and $120 \mathrm{kVp}$ group while all other scanning parameters were kept constant. Signal-to-noise ratio (SNR) and contrast-to-noise ratio (CNR) were calculated for locations along the carotid artery, vertebral artery and circle of Willis. Three radiologists independently assessed subjective image quality including confidence of visualization using a five-point scale. Effective dose was calculated using the dose length product (DLP). CNR and SNR were either similar or better when using $100 \mathrm{kVp}$ for all vessels. Subjective image quality was similar between techniques. Mean effective dose was 36\% lower for the 100 $\mathrm{kVp}$ than the $120 \mathrm{kVp}$ technique. This study demonstrated that using $100 \mathrm{kVp}$ technique provides $36 \%$ dose reduction with significantly higher objective image quality and nonsignificantly higher subjective image quality compared to the $120 \mathrm{kVp}$ technique.
\end{abstract}

Keywords Multidetector Computed Tomography, Computed Tomography Angiography, Carotid arteries, Radiation, Radiation dosage

\section{Introduction}

Stroke is the third leading cause of death and is a major source of disability in Canada [1]. Evaluation of the carotid arteries for occlusive disease is standard amongst patients with ischemic stroke. Carotid computed tomography angiography (CTA) is rapid, non-invasive, and a reliable method of evaluating the carotid arteries with easy accessibility at many Canadian centres. Other non-invasive imaging modalities are available, such as doppler carotid sonography and magnetic resonance angiography (MRA) [2]. Although both MRI and ultrasound benefit from having no ionizing radiation, there are limitations compared with CT.
Carotid doppler ultrasound is quite sensitive for significant stenosis, but is less specific than CTA, has high operator variability and does not allow assessment of the origin of the supra-aortic or intracranial arteries [2,3]. Contrast enhanced MRA has better performance than non-contrast Time-of Flight [2], with associated increasing cost and imaging time of the procedure. Also, MRA is not easily accessible in many institutions, has long acquisition times, and is contraindicated in patients with claustrophobia, medical implants, and metallic foreign bodies.

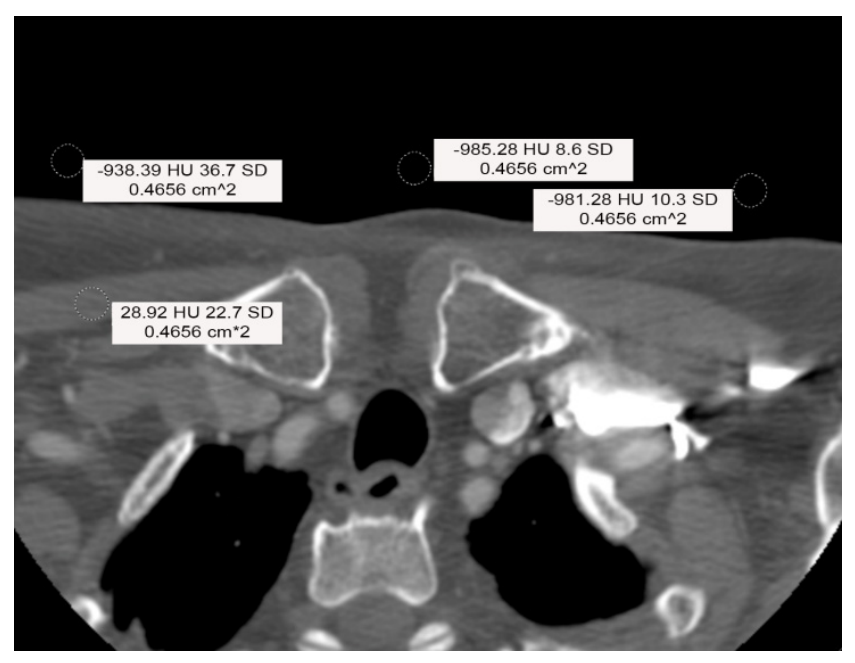

Figure 1. Show the Styles window

Figure 1: 79-year-old women in the $120 \mathrm{kV}$ group demonstrating background noise and muscle SI measurement for the supra-aortic common carotid and vertebral arteries.

One major disadvantage of CTA is exposure of ionizing radiation and the associated risk of radiation-induced cancers [4]. It is estimated that $1.5 \%$ to $2 \%$ of cancers in the United States are attributable to ionizing radiation from diagnostic CT [4]. With the development of newer CT technology in the last decade, the number of CT examinations and the radiation dose per examination has increased substantially [4]. This has led to a calling for 
strategies to decrease the exposure of radiation to patients. Tube current (mA) adjustment based on patient body size or body part has been one strategy to decrease radiation dose. Tube potential $(\mathrm{kV})$ has generally been standardized for a given clinical application, however some recent studies have shown that lowering tube potential can be an effective way of reducing radiation dose in CTA without compromising image quality [5].

The purpose of this study is to compare radiation dose and both subjective and objective image quality using the standard $120 \mathrm{kVp}$ technique and the lower dose $100 \mathrm{kVp}$ technique for both carotid and circle of Willis CT angiography and discuss its feasibility in every day clinical practice.

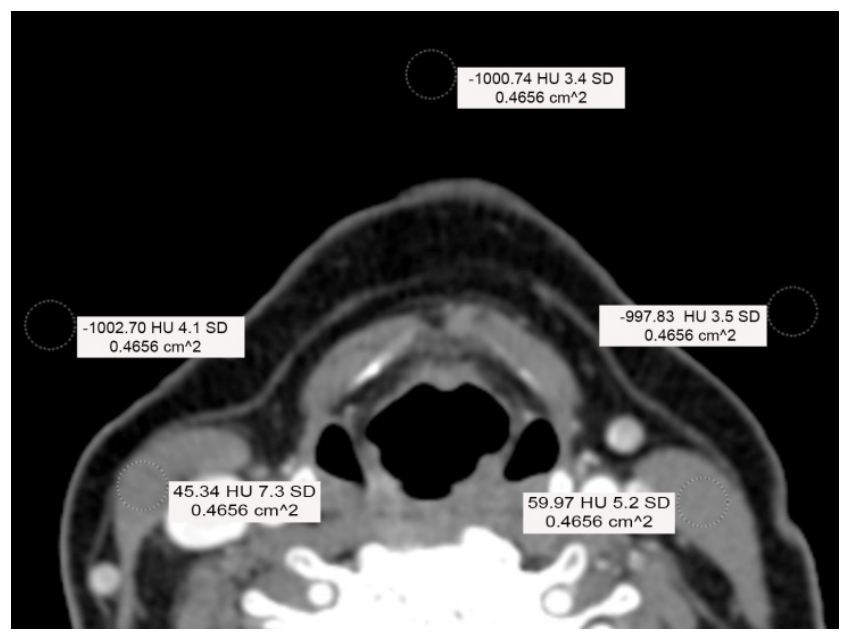

Figure 2. Manage Styles $\rightarrow$ Import/Export

Figure 2: 60 -year-old in the $100 \mathrm{kVp}$ group demonstrating background noise and muscle SI measurements for the CCA at the level of the thyroid cartilage, bulb, ICA, ECA and cOW.

\section{Materials and Methods}

\subsection{Patient Population}

Ethics approval was obtained from the University of Saskatchewan. Biomedical Research Ethics Board and informed consent was obtained from all participants. Health Insurance Portability and Accountability Act (HIPAA) regulations were followed. All non-pregnant adult patients referred for carotid stenosis assessment with carotid CT angiography between May 2009 and August 2009 at our institution were prospectively added to this study. Patients were excluded from the study if they had contraindications to CT angiography or consent was denied.

Age, height, and weight were recorded at the time of the scan. Body mass index (BMI) was calculated as patient body weight $(\mathrm{kg})$ divided by the square of height $\left(\mathrm{m}^{2}\right)$. Neck size was assessed for each patient on a GE Advantage Workstation, Version 4.4 (GE Medical Systems, Wisconsin) by measuring neck circumference, AP distance at midline, and transverse distance at the widest part of the neck at the level of the hyoid bone.

\subsection{Carotid CT Angiography Protocol}

All scans were performed using a GE LightSpeed Ultra eight channel scanner (GE Medical Systems, Wisconsin). Anterior-to-posterior and lateral scout views were acquired at $120 \mathrm{kVp}$ and $10 \mathrm{~mA}$. The patients were examined in the supine position in the craniocaudal direction with scan coverage from the frontal sinuses to the aortic arch allowing for inclusive assessment of the circle of Willis and carotid systems. Consecutive patients were alternated between the $100 \mathrm{kVp}$ group (group $1, \mathrm{n}=20$ ) and the $120 \mathrm{kVp}$ group (group 2, $\mathrm{n}=20$ ). All other scanning parameters were kept constant between groups. Scan-field-of-view of $50 \mathrm{~cm}$ and display field of view of $18.0 \mathrm{~cm}$ was used, with a table speed of $13.5 \mathrm{~mm} / \mathrm{sec}$, gantry rotation speed of 0.7 seconds and a pitch factor of 1.35:1. Fixed tube current (266 mAs) was used. $100 \mathrm{cc}$ of Optiray 320 (Tyco Healthcare, Montreal, Quebec) IV contrast were automatically injected at 3 cc/second using a Medrad envision single chamber power injector (Medrad Inc, Indianola, Pennsylvania).

\subsection{CT Dose Assessment}

Volume CT dose index $\left(\mathrm{CTDI}_{\mathrm{vol}}\right)$, scan length, and dose length product (DLP) were recorded from the scanner for each participant. To calculate effective dose, DLP was multiplied by $0.0054 \mathrm{mSv} / \mathrm{mGy} \cdot \mathrm{cm}$, a conversion factor specific for neck CT scans [6].

\subsection{Vessel Analysis}

Assessment of image quality was performed according to the methods of Heyer et al [7]. Review was performed on a Philips iSite Radiology PACS system software version 3.6.81 (Enterprise Imaging, Philips Healthcare, Foster City, CA) by a single author (Blinded). Objective image quality was evaluated by measuring signal intensity (SI) in Hounsfield units (HUs) of regions of interest (ROIs) along the right and left carotid systems at consistent anatomic landmarks of different locations: the supra-aortic common carotid artery (SA CCA) $1 \mathrm{~cm}$ above the origin of the artery, the common carotid artery (CCA) at the level of the thyroid cartilage, internal carotid artery (ICA) $1 \mathrm{~cm}$ above the bifurcation, external carotid artery (ECA) $1 \mathrm{~cm}$ above the bifurcation, and the carotid bulb. The vertebral arteries were assessed at two locations: the supra-aortic vertebral artery (SA VERT) $1 \mathrm{~cm}$ above the origin of the artery and the vertebral artery (VERT) at the level of the thyroid cartilage. SI measurements for the circle of Willis were assessed at three locations: the right and left internal carotid artery (COW ICA), the basilar artery (BA) measured twice corresponding to the slice at which the right and left ICA measurements were taken, and the right and left middle 
cerebral artery (MCA). Circular ROIs were positioned to sample as much cross sectional area of vessel as possible without inclusion of vessel wall or plaque. The carotid bulb and middle cerebral artery was measured using a freehand ROI.

Background noise (BN) for the supra-aortic arteries (SA CCA and SA VERT) was measured as the average standard deviation (in HUs) from surrounding air at three ROIs in front of the patient (right, left and center) at the level of the clavicular heads (Fig. 1). Muscle SI was measured at this level in the pectoralis major muscle on the side opposite contrast injection. Background noise for the remaining arteries was measured as the average standard deviation (in HUs) from surrounding air at three ROIs in front of the patient (right, left and center) at the level of the aryepiglottic folds (Fig. 2). Muscle SI was measured at this level in the right and left sternocleidomastoid muscle. BN and muscle SI were measured with a standard circular size of $0.5 \mathrm{~cm}^{2}$ at all locations. Background noise measurements that were two standard deviations away from the mean were excluded (3 measurements from the $120 \mathrm{kVp}$ group and 2 measurements from the $100 \mathrm{kVp}$ group all at the level of the aryepiglottic folds) and the remaining measurements were averaged.
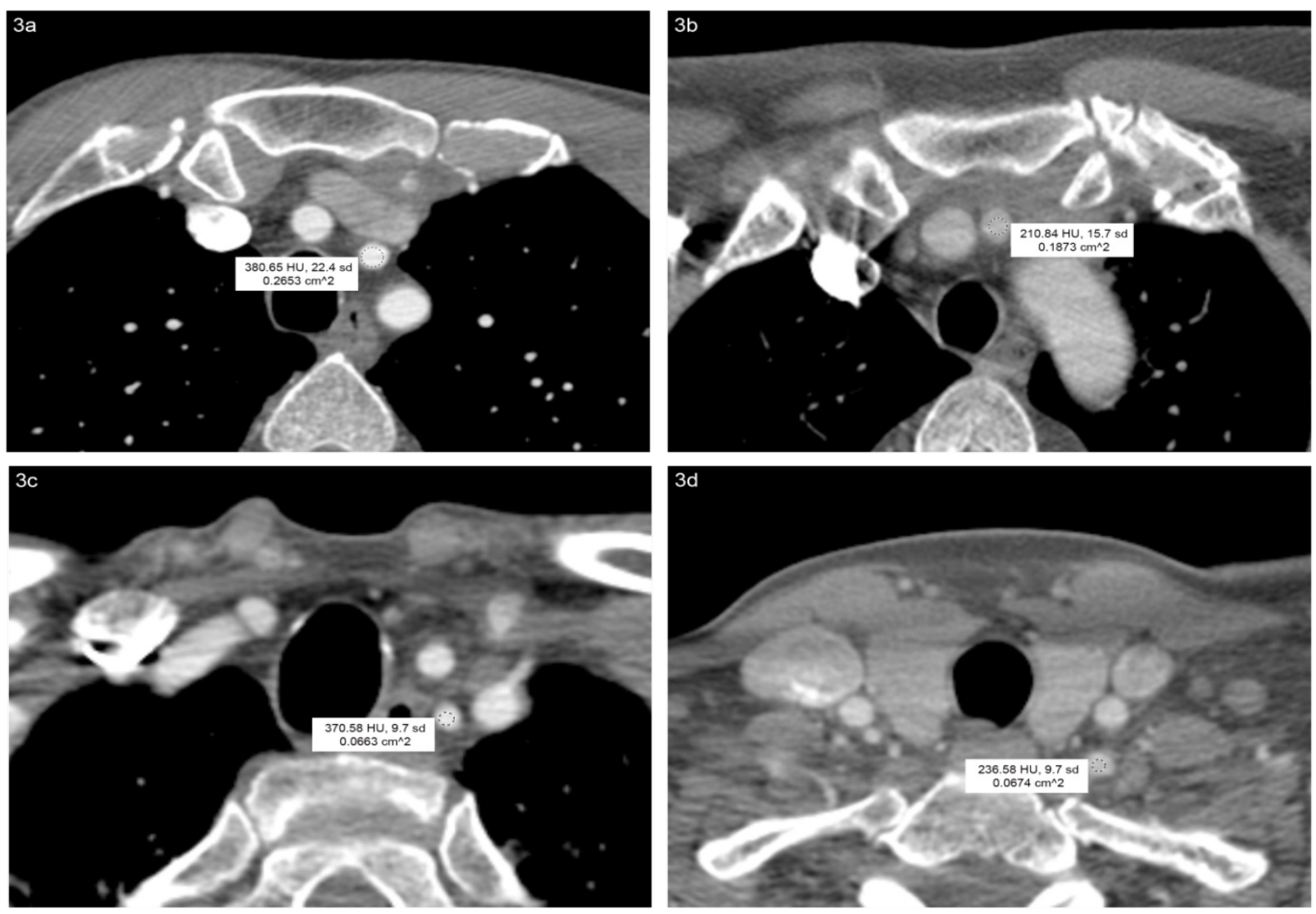

Figure 3. (A,C) 55-year-old male patient with CTA using $100 \mathrm{kVp}$ and (B,D) 41 -year-old male patient with CTA using $120 \mathrm{kVp}$. Effective doses were $2.33 \mathrm{mSv}(100 \mathrm{kVp}$ patient in A,C) and $3.27 \mathrm{mSv}$ (120 kVp patient in B,D). CT numbers and standard deviations were measured in Hounsfield Units (HU). In A and B, SNR (22.6 vs 15.1) and CNR (18.5 vs 12.8) measurements of the left supra-aortic common carotid arteries located $1 \mathrm{~cm}$ above the origin of the artery. In C and D, SNR (22.1 vs 17.0$)$ and CNR (18.0 vs 14.7) measurements for the supra-aortic vertebral artery $1 \mathrm{~cm}$ above the origin of the artery. Windowing and levels are constant in both patients 

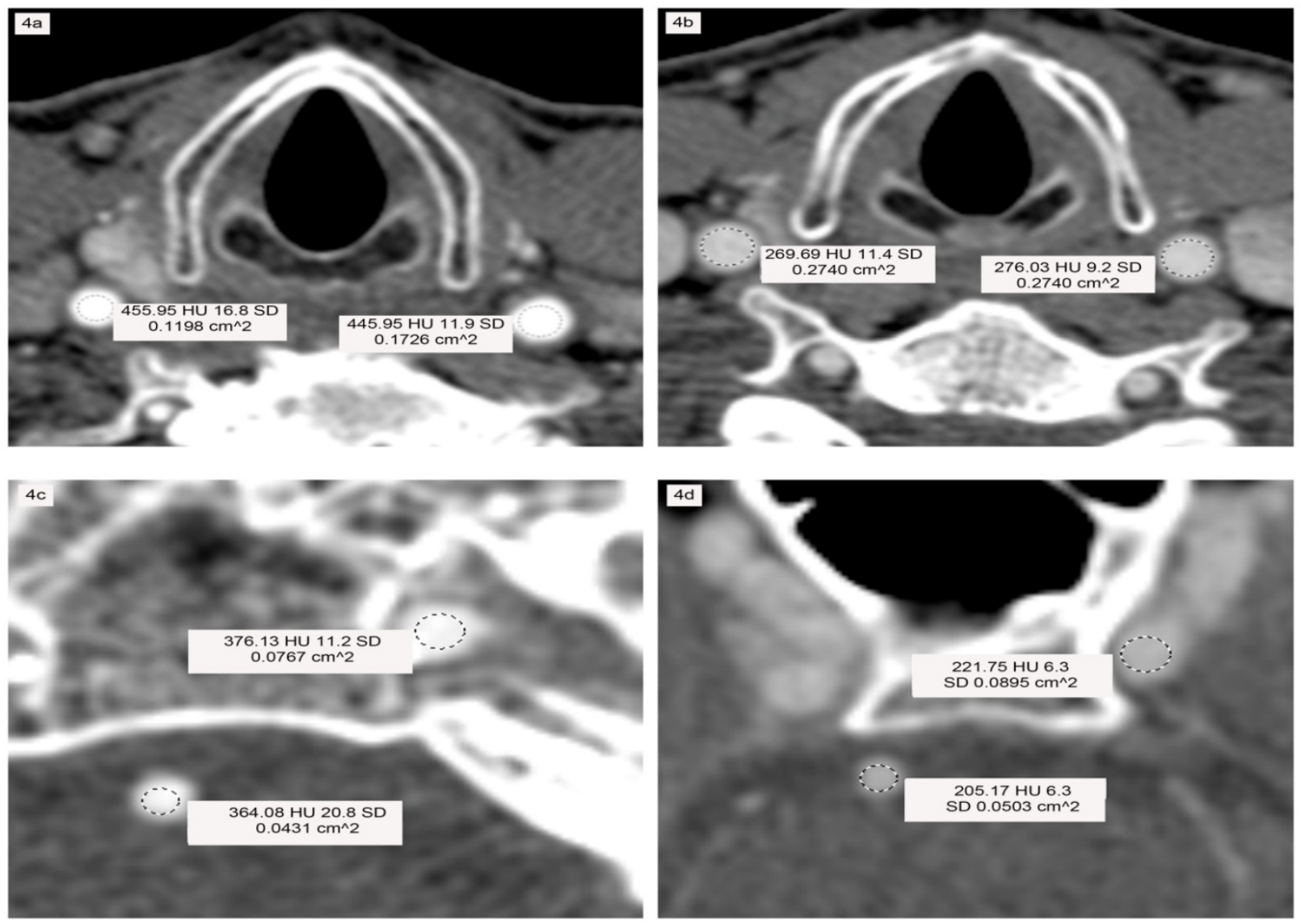

Figure 4. (A,C) 76-year-old male patient with CTA using $100 \mathrm{kVp}$ and (B,D) a 47 -year-old male with CTA using $120 \mathrm{kVp}$. Effective doses were $2.26 \mathrm{mSv}$ $(100 \mathrm{kVp}$ patient in $\mathrm{A}, \mathrm{C})$ and $3.60 \mathrm{mSv}(120 \mathrm{kVp}$ patient in B,D). CT numbers and standard deviations were measured in Hounsfield Units (HU). In A and B, SNR (98.0 vs 71.2) and CNR (86.3 vs 56.0) measurements of the right and left common carotid arteries at the level of the thyroid cartilage. In C and D, SNR (81.7 vs 57.8) and CNR (70.9 vs 43.0) measurements for the circle of Willis internal carotid artery and SNR (77.3 vs 52.6) and CNR (65.6 vs 37.4$)$ measurements for the basilar artery in the head. Windowing and levels are constant in both patients

Table 1. Patient Characteristics

\begin{tabular}{|c|c|c|c|}
\hline Patient Characteristics & $100 \mathrm{kVp}$ group & $120 \mathrm{kVp}$ group & P-value $^{\dagger}$ \\
\hline Age (years) & $62 \pm 23$ & $63 \pm 16$ & 0.84 \\
\hline Male-to-Female Ratio & $7: 13$ & $9: 11$ & 0.08 \\
\hline Neck Size (AP) (mm) & $155.5 \pm 21.3$ & $167.6 \pm 29.0$ & 0.34 \\
\hline Neck Size (trx) (mm) & $126.0 \pm 13.4$ & $131.5 \pm 19.5$ & 0.11 \\
\hline Neck Size (Circ) $(\mathrm{mm})$ & $451.4 \pm 54.0$ & $478.4 \pm 51.6$ & 0.90 \\
\hline BMI (kg/m $\left.{ }^{2}\right)$ & $28.3 \pm 4.8$ & $28.1 \pm 5.0$ & 2 \\
\hline
\end{tabular}

Note $-{ }^{\dagger}$ t-test, $\mathrm{AP}=$ Anterior-posterior, $\operatorname{trx}=$ transverse, circ $=$ circumference, $\mathrm{mm}=$ millimeters, $\mathrm{BMI}=$ Body mass index, $\mathrm{N} / \mathrm{A}=$ not applicable. 
Table 2. Mean Signal to Noise and Contrast to Noise Ratios (SNR and CNR) with Standard Deviation for the $100 \mathrm{kVp}$ and $120 \mathrm{kVp}$ Groups

\begin{tabular}{|c|c|c|c|c|}
\hline Vessel & Measure & $100 \mathrm{kVp}$ & $120 \mathrm{kVp}$ & P-value ${ }^{\dagger}$ \\
\hline \multirow{2}{*}{ SA CAA } & SNR & $22.7( \pm 9.6)$ & $20.2( \pm 11.3)$ & $0.29(0.146)$ \\
\hline & CNR & $19.8( \pm 8.5)$ & $16.9( \pm 10.0)$ & $0.17(0.081)$ \\
\hline \multirow{2}{*}{$\mathrm{CCA}$} & SNR & $70.7( \pm 43.5)$ & $51.6( \pm 34.6)$ & $0.03(0.044)$ \\
\hline & $\mathrm{CNR}$ & $59.4( \pm 37.7)$ & $41.1( \pm 27.9)$ & $0.02(0.03)$ \\
\hline \multirow{2}{*}{ Bulb } & SNR & $71.7( \pm 41.7)$ & $54.6( \pm 35.7)$ & $0.05(0.059)$ \\
\hline & $\mathrm{CNR}$ & $60.4( \pm 36.0)$ & $44.1( \pm 29.1)$ & $0.03(0.049)$ \\
\hline \multirow{2}{*}{ ICA } & SNR & $67.5( \pm 40.5)$ & $54.4( \pm 35.8)$ & $0.13(0.111)$ \\
\hline & CNR & $56.4( \pm 34.5)$ & $43.9( \pm 29.2)$ & $0.09(0.111)$ \\
\hline \multirow{2}{*}{ ECA } & SNR & $69.2( \pm 41.3)$ & $53.4( \pm 35.4)$ & $0.07(0.065)$ \\
\hline & $\mathrm{CNR}$ & $57.9( \pm 35.5)$ & $42.9( \pm 28.8)$ & $0.04(0.04)$ \\
\hline \multirow{2}{*}{ SA VERT } & SNR & $21.6( \pm 8.6)$ & $20.1( \pm 9.5)$ & $0.47(0.370)$ \\
\hline & $\mathrm{CNR}$ & $19.2( \pm 7.6)$ & $16.7( \pm 8.2)$ & $0.17(0.117)$ \\
\hline \multirow{2}{*}{ VERT } & SNR & $68.6( \pm 43.4)$ & $51.2( \pm 33.7)$ & $0.05(0.058)$ \\
\hline & CNR & $60.3( \pm 37.8)$ & $44.0( \pm 30.1)$ & $0.04(0.050)$ \\
\hline \multirow{2}{*}{ ICA (COW) } & SNR & $57.4( \pm 35.2)$ & $44.0( \pm 28.7)$ & $0.07(0.096)$ \\
\hline & CNR & $46.1( \pm 29.6)$ & $33.5( \pm 22.0)$ & $0.03(0.06)$ \\
\hline \multirow{2}{*}{$\mathrm{MCA}$} & SNR & $50.2( \pm 32.3)$ & $39.0( \pm 25.5)$ & $0.09(0.122)$ \\
\hline & CNR & $38.9( \pm 26.6)$ & $28.5( \pm 18.8)$ & $0.05(0.082)$ \\
\hline \multirow{2}{*}{ Bas } & SNR & $54.4( \pm 34.2)$ & $41.1( \pm 26.9)$ & $0.06(0.081)$ \\
\hline & CNR & $43.6( \pm 28.4)$ & $30.6( \pm 20.0)$ & $0.02(0.036)$ \\
\hline
\end{tabular}

Note - ICA $=$ internal carotid artery, ECA $=$ external carotid artery, SA VERT $=$ supraaortic vertebral artery, VERT $=$ vertebral artery, COW= circle of Willis, MCA = middle cerebral artery

*T-test and Wilcoxon rank sum test in parenthesis

Table 3. Subjective Image Quality Scores for Four Locations along the Carotid Artery and Confidence of Visualization Rated on a 5-Point Scale as Judged by Three Independent Reviewers

\begin{tabular}{|c|c|c|c|c|c|c|c|c|}
\hline & $\mathrm{kVp}$ & $\mathrm{n}$ & 1 & 2 & 3 & 4 & 5 & $\operatorname{Avg}_{W}{ }^{*}$ \\
\hline \multirow{2}{*}{$\mathrm{CCA}$} & 100 & 0 & 0 & 0 & 5 & 49 & 66 & 4.51 \\
\hline & 120 & 0 & 0 & 0 & 5 & 53 & 62 & 4.48 \\
\hline \multirow{2}{*}{ Bulb } & 100 & 0 & 0 & 0 & 4 & 54 & 62 & 4.48 \\
\hline & 120 & 0 & 0 & 0 & 6 & 53 & 61 & 4.46 \\
\hline \multirow{2}{*}{ ICA } & 100 & 0 & 0 & 0 & 0 & 50 & 64 & 4.56 \\
\hline & 120 & 0 & 0 & 0 & 6 & 53 & 61 & 4.46 \\
\hline \multirow{2}{*}{ ECA } & 100 & 0 & 0 & 0 & 1 & 63 & 56 & 4.46 \\
\hline & 120 & 0 & 0 & 0 & 3 & 63 & 54 & 4.43 \\
\hline \multirow{2}{*}{$\begin{array}{l}\text { Confidence of } \\
\text { Visualization }\end{array}$} & 100 & 0 & 0 & 0 & 0 & 45 & 72 & 4.62 \\
\hline & 120 & 0 & 0 & 0 & 9 & 40 & 71 & 4.52 \\
\hline
\end{tabular}

Note $-\mathrm{CCA}=$ common carotid artery, Bulb = carotid bulb, ICA = internal carotid artery, ECA = external carotid artery, *Weighted average 


\subsection{Statistical Analysis}

In the objective component of our study, the primary outcomes are SNR and CNR. For both SNR and CNR: assuming a difference of 15 and standard deviation of 15 between the $100 \mathrm{kVp}$ and $120 \mathrm{kVp}$ techniques, a total of 34 patients (17 patients per group) would be needed to demonstrate statistical difference $(p$-value $<0.05)$ with $80 \%$ power using a two-tailed t-test. Our study used a total of 40 participants. Descriptive statistics was used to summarize the data. To compare differences between two groups, a Student's t-test and the Wilcoxon rank sum test was used for continuous variables. A $p$-value of less the 0.05 was considered statistically significant. Analyses were carried out with SAS statistical software version 9.2 (The SAS Institute, Cary, North Carolina).

\section{Results}

Comparison of patient characteristics between both groups did not reveal any significant differences for age, sex, BMI, and neck size and therefore substantiates the validity of our results for subjective and objective measurements (Table $1)$.

During objective measurements, two right-sided ICA measurements were excluded from the $100 \mathrm{kVp}$ group due to one complete occlusion and one stent. The case with complete occlusion also excluded the right ICA COW) and the right MCA measurements. Also from this group, there was one complete occlusion of one left-sided vertebral artery excluding measurements at both the supra-aortic and thyroid cartilage levels. From the $120 \mathrm{kVp}$ group, three right-sided vertebral arteries

were completely occluded and measurements were excluded at both the supra-aortic and thyroid cartilage levels for two of these arteries and one was excluded at the level of the thyroid cartilage only.

CNR and SNR were similar or better for all vessels when using the $100 \mathrm{kVp}$ technique (Table 2). This was significant for CCA SNR and CNR, bulb CNR, ECA CNR, VERT CNR, ICA (COW) CNR, and basilar artery CNR. Analyzing all vessels as a group, the $100 \mathrm{kVp}$ group had significantly higher SNR (55.4 \pm 39.3 vs. $43.0 \pm 31.2, p<0.001$ with t-test and 0.0001 with Wilcoxon rank sum test) and CNR (46.2 \pm 33.5 vs. $34.2 \pm 25.6, p<0.001$ with t-test and 0.0001 with Wilcoxon rank sum test). The mean background noise was not significantly different for the $100 \mathrm{kVp}$ and $120 \mathrm{kVp}$ groups (16.3 SDHU \pm 8.4 SDHU and 13.6 SDHU \pm 24.1 SDHU, respectively; $p=0.4$ ).

During subjective assessment, two (cumulative total of 6) ICAs from the $100 \mathrm{kVp}$ group were not assessed because of one stent and one complete occlusion. The stent case was also excluded from the general confidence of visualization assessment. The weighted average score for subjective image quality of the ICA was 4.56 for the $100 \mathrm{kVp}$ technique and 4.46 for the $120 \mathrm{kVp}$ technique (Table 3). Higher mean scores were also found in the CCA, bulb and ECA. For confidence of visualization, two of the three reviewers rated the $100 \mathrm{kVp}$ group nonsignificantly higher overall. When combining scores from all three reviewers, $100 \%$ of $100 \mathrm{kVp}$ and $93 \%$ of $120 \mathrm{kVp}$ exams were rated as either 'very confident' or 'good confidence' (Table 3).

Between the $100 \mathrm{kVp}$ technique and the $120 \mathrm{kVp}$ technique, there was no significant difference in scan length

$(287.36 \mathrm{~mm} \pm 20.3$ vs $280.64 \mathrm{~mm} \pm 27.4$, respectively; $p=$ 0.38). There was a significant difference in $\mathrm{CTDI}_{\mathrm{vol}}(12.82$ $\mathrm{mGy} \pm 0.62$ vs $20.77 \mathrm{mGy} \pm 1.53$, respectively; $p<.001)$ and DLP $(399.5 \mathrm{mGy} \cdot \mathrm{cm} \pm 64.4$ vs $622.8 \mathrm{mGy} \cdot \mathrm{cm} \pm 40.3$, respectively; $p<.001$ ). Mean effective dose was $36 \%$ lower for the $100 \mathrm{kVp}$ than the $120 \mathrm{kVp}$ technique $(2.15 \pm 0.35$ $\mathrm{mSv}$ vs. $3.36 \pm 0.22 \mathrm{mSv}, p<0.001)$.

\section{Discussion}

Image quality and diagnostic confidence is often sacrificed when applying strategies for lowering radiation dose because image noise increases. However, lowering kilovoltage closer to the k-edge of iodine both decreases dose while increasing vessel attenuation during CTA. SNR and CNR are commonly used objective measurements for image quality in CT angiography because they take into account both vessel enhancement and image noise. Our study showed an overall significantly higher SNR and CNR for the $100 \mathrm{kVp}$ group than the $120 \mathrm{kVp}$ group in both the carotid and circle of Willis while background image noise was non-significantly higher in the $100 \mathrm{kVp}$ group.

The attenuation (density) of a material scanned during CT is greater for those that absorb more photons during the scan. Better matching of tube voltage to the k-edge of a material results in greater photon absorption, and therefore, greater attenuation on the CT images. The effective $\mathrm{kV}$ of a beam is one-third to one-half the $\mathrm{kVp}$ of the beam (7), 33-50 keV for the $100 \mathrm{kVp}$ technique and $40-60 \mathrm{KeV}$ for the $120 \mathrm{kVp}$ technique. Therefore, the $100 \mathrm{kVp}$ technique better matches the $33 \mathrm{kEv}$ k-edge of iodine (8).

Our results for subjective image quality are in keeping with our objective results. Since carotid CTA is most commonly performed to search for stenosis or occlusion, we feel that confidence of visualization was the most important subjective assessment performed. This included the ability to assess the arterial wall, carotid plaque and calcification. There was a non-significant better confidence of visualization for the $100 \mathrm{kVp}$ group. Even though subjective image quality assessment was focused on the carotid arteries, the objective data from the origin of the common carotids, vertebral arteries and circle of Willis show that $100 \mathrm{kVp}$ image quality is similar or better throughout the regions assessed during clinical carotid, vertebral, and circle of Willis CT angiography.

Our study demonstrated a $36 \%$ reduction in effective dose using the $100 \mathrm{kVp}$ technique. This decrease is important considering the accumulation of dose with repeated imaging, 
the large span of body volume being imaged (which includes the particularly radiosensitive thyroid and eye lenses), and the wide age range of the population receiving CTA of the carotids (22 to 90 in our study).

To our knowledge, no study to date has looked at both carotid and circle of Willis CTA at $100 \mathrm{kVp}$. A previous study by Beitzke et al examined reduced tube voltage in the supra-aortic arteries including carotids, vertebrals and COW using $120 \mathrm{kVp}$ or $80 \mathrm{kVp}$ with a fixed tube current of 300 $\mathrm{mAs}$ on a 64 detector row scanner [9]. The $80 \mathrm{kVp}$ had $69 \%$ lower radiation dose than the $120 \mathrm{kVp}$ technique [9]. The 80 $\mathrm{kVp}$ had higher SNR and CNR at the level of the ICA [9]. The $80 \mathrm{kVp}$ group also showed significantly higher background noise and they noted that some $80-\mathrm{kVp}$ examinations showed poor diagnostic image quality in their subjective assessment. They attributed this to streak artifact from the remaining contrast in the brachiocephalic veins occurring more commonly in those with above average BMI [9]. Background noise findings and lower subjective image quality at $80 \mathrm{kVp}$ are in contrast to our study's $100 \mathrm{kVp}$ technique, which demonstrates better subjective image quality overall and all were considered diagnostic. Furthermore, patients presenting with signs and symptoms of ischemic TIA or stroke will often have a simultaneous examination of their carotid and cerebral arteries. The thoracic aperture, neck and head areas are characterized by major differences in attenuation and although a lower tube voltage of $80 \mathrm{kVp}$ may be sufficient in the extracranial carotid arteries, one study did demonstrate that without adjusting tube current, it does not provide adequate image quality in the head [10].

Two previous studies investigating reduced tube voltage in cerebral CTA have also showed significant dose reduction while maintaining image quality. Waaijer et al compared 120 $\mathrm{kVp}$ with $200 \mathrm{mAs}$ versus $90 \mathrm{kVp}$ with $330 \mathrm{mAs}$ for CTA of the circle of Willis [11]. Results from this study showed a 30\% reduction in dose with a decrease in kilovoltage of $30 \mathrm{kVp}$ $(120$ vs. $90 \mathrm{kVp})$ [11]. This dose reduction is less than our $36 \%$ dose reduction from $20 \mathrm{kVp}$ drop (120 vs. $100 \mathrm{kVp}$ ) because of a Waaijer et al's compensatory increase in tube current. The Waaijer et al $90 \mathrm{kVp}$ group did show significantly better subjective attenuation and better objective assessment of image quality in the intracranial ICA, with no difference in image noise [11]. Cho et al. compared $120 \mathrm{kVp}$ and $150 \mathrm{mAs}$ with high concentration contrast material and $80 \mathrm{kVp}$ and $370 \mathrm{mAs}$ with moderate concentration contrast material [12]. The $80 \mathrm{kVp}$ group showed $22 \%$ dose reduction, again less than our $36 \%$ because of the compensatory increased tube current. Cho et al's $80 \mathrm{kVp}$ group also showed higher vessel attenuation, SNR, CNR and better subjective assessment of image quality - despite higher background noise [12]. Although we also showed higher CNR in all intracranial vessels assessed, it is difficult to directly compare results of both of these studies to our data because of the compensatory increase in tube current used [11,12].

When modifying CT techniques, it is important to keep in mind automatic tube current modulation (ATCM). In a study of 78 patients, Namasivayam et al showed $21-33 \%$ dose reduction using z-axis ATCM with $120 \mathrm{kVp}$ tube current during contrast-enhanced scanning of the neck [13]. Although objective noise was increased, the ATCM images were found to have acceptable image noise and adequate diagnostic acceptability [13]. A study of 50 patients receiving craniocervical CT Angiography by Lee et al demonstrated an $18 \%$ dose reduction using combined ATCM technique at $120 \mathrm{kVp}$ [14]. Although objective image quality was increased, they found no difference in subjective image quality [14].

Given that ATCM systems modulate tube current $(\mathrm{mA})$ to maintain a given image quality (typically determined as a level of noise), ATCM systems would be expected to increase tube current to maintain image quality as noise is expected to increase with lower $\mathrm{kVp}$ technique. It should, however be noted that background noise was similar for both groups (12.3 SDHU $\pm 29.0 \mathrm{SDHU}$ for $100 \mathrm{kVp}$ group and 13.6 SDHU $\pm 24.1 \mathrm{SDHU}$ for $120 \mathrm{kVp}$ group) in our study. Our finding of similar noise with lower tube potential is in keeping with a previous phantom study showing that image noise is held relatively constant when lowering $\mathrm{kV}$ for smaller phantoms, with the inverse relationship between image noise and $\mathrm{kV}$ only seen for larger phantoms [5].

There are limitations to our study. As in the Lee et al study, different patients were exposed to the different techniques, as it would not have been possible to expose the same patients to the two different techniques. This is because of obvious concerns for radiation dose and increased contrast load from repeating the study. We also chose to use alternating assignments to the groups as opposed to random assignment to ensure equal numbers were in each group. The method of alternating patients between groups did not affect the order in which patients were referred for carotid CT angiography and therefore we believe it did not affect assigned technique. Although this is a relatively small sample size, we did achieve a statistical power of $80 \%$. Additionally, there was a trend towards better image quality with the lower dose group. We did not recruit any further patients for analysis, as we could not justify continuing to expose patients to the higher radiation doses associated with the $120 \mathrm{kVp}$ technique. Our institution now uses $100 \mathrm{kVp}$ for carotid CT angiography. Additionally, we concentrated on objective image quality including SNR and CNR as well as confidence of visualization with no direct assessment of clinical features such as degree of stenosis.

\section{Conclusion}

Utilizing $100 \mathrm{kVp}$ vs $120 \mathrm{kVp}$ technique for Carotid CT Angiography provided a significant dose reduction of $36 \%$, with significantly higher objective image quality and nonsignificantly higher subjective image quality.

\section{Acknowledgements}


The authors of this study would like to acknowledge the University of Saskatchewan College of Medicine Dean's Project Summer Research Program and the University of Saskatchewan/Saskatoon Health Region Medical Imaging Continuing Research Fund for funding Patricia Jo's salary and poster presentation expenses. Their financial support is very much appreciated. We would also like to thank the Department of Medical Imaging Resident Research Day for the medical student prize, the Canadian National Medical Student Research Symposium for $3^{\text {rd }}$ prize in the clinical research category and the Canadian Association of Radiologists for awarding us with the top scientific poster prize at the $73^{\text {rd }}$ Annual Scientific Meeting.

We would also like to thank Lori Toews, Troy Anderson and the other participating CT technologists. We are very grateful for their time and dedication to the project and without their help, this would not have been possible.

\section{REFERENCES}

[1] Statistics Canada, CANSIM table 102-0561 and Catalogue no. 84-215-X. Last modified: 2011-11-01.

[2] M. Gough. Preprocedural imaging strategies in symptomatic carotid artery stenosis. Journal of Vascular Surgery,Vol. 54, No. 4, 1215-1218, 2011

[3] J. U-King-Im, V. Young, J. Gillard. Carotid-artery imaging in the diagnosis and management of patients at risk of stroke. Lancet Neurology, Vol. 8, No. 6, 569-580, 2009.

[4] D. Brenner, E. Hall. Computed Tomography: An Increasing Source of Radiation Exposure. New England Journal of Medicine, Vol. 357, 2277-2284, 2007.

[5] C. McCollough, A. Primak, N. Braun, J. Kofler, L. Yu, J. Christner. Strategies for Reducing Radiation Dose in CT. Radiology Clinics of North America, Vol. 47, No. 1, 27-40, 2009.
[6] W. Huda, K. Ogden, M. Khorasani. Converting dose-length product to effective dose at CT. Radiology, Vol. 248, 995-1003, 2008.

[7] C. Heyer, P. Mohr, S. Lemburg, S. Peters, V. Nicolas. Image quality and radiation exposure at pulmonary $\mathrm{CT}$ angiography with 100- or 120- protocol: prospective randomized study. Radiology, Vol. 245, No. 2, 577-583, 2007.

[8] W. Huda. Review of Radiological Physics. Lippincott Williams \& Wilkins, Philadelphia, 2010.

[9] D. Beitzke, F. Wold, G. Eddhause, C. Plank, R. Schernthaner, M. Weber, R. Nolz, J. Lammer, C. Loewe. Computed tomography angiography of the carotid arteries at low $\mathrm{kV}$ settings: a prospective randomized trial assessing radiation dose and diagnostic confidence. Eurpean Radiology, Vol. 21, No. 11, 2434-2444, 2011.

[10] B. Ertl-Wagner, R. Hoffmann, R. Bruning, K. Herrmann, B. Snyder, J. Blume, M. Reiser. Multi-detector row CT angiography of the brain at various kilovoltage settings. Radiology, Vol. 231. No. 2, 528-535, 2004.

[11] A. Waaijer, M. Prokop, B. Velthuis, C. Bakker, G. de Kort, M. van Leeuwen. Circle of Willis at CT angiography: dose reduction and image quality - reducing tube voltage and increasing tube current settings. Radiology, Vol. 242. No. 3, 832-839, 2007.

[12] E. Cho, T. Chung, D. Oh, H. Choi, S. Suh, H. Lee, K. Lee. Cerebral computed tomography angiography using a low tube voltage $(80 \mathrm{kVp})$ and a moderate concentration of iodine contrast material. Investigative Radiology, Vol. 47. No. 2, 142-147, 2012.

[13] S. Namasivayam, M. Kalra, K. Pottala, S. Waldrop, P. Hudgins. Optimization of Z-axis automatic exposure control for multidetector row CT evaluation of neck and comparison with fixed tube current technique for image quality and radiation dose. American Journal of Neuroradiology, Vol. 27. No. 10, 2221-2225, 2006.

[14] E. Lee, S. Lee, R. Agid, J. Bae, K. terBrugge. Comparison of image quality and radiation dose between fixed tube current and combined automatic tube current modulation in craniocervical CT angiography. American Journal of Neuroradiology, Vol. 30, No. 9, 1754-1759, 2009. 